\title{
Magnetic field dependence of dephasing rate due to diluted Kondo impurities
}

\author{
T. Micklitz, ${ }^{1}$ T. A. Costi, ${ }^{2}$ and A. Rosch ${ }^{1}$ \\ ${ }^{1}$ Institute for Theoretical Physics, University of Cologne, 50937 Cologne, Germany \\ ${ }^{2}$ Institut für Festkörperforschung, Forschungszentrum Jülich, 52425 Jülich, Germany
}

(Received 13 October 2006; published 9 February 2007)

\begin{abstract}
We investigate the dephasing rate, $1 / \tau_{\varphi}$, of weakly disordered electrons due to scattering from diluted dynamical impurities. Our previous result for the weak-localization dephasing rate is generalized from diluted Kondo impurities to arbitrary dynamical defects with typical energy transfer larger than $1 / \tau_{\varphi}$. For magnetic impurities, we study the influence of magnetic fields on the dephasing of Aharonov-Bohm oscillations and universal conductance fluctuations both analytically and using the numerical renormalization group. These results are compared to recent experiments.
\end{abstract}

DOI: 10.1103/PhysRevB.75.054406

PACS number(s): 72.15.Lh, 72.15.Qm, 72.15.Rn

\section{INTRODUCTION}

Decoherence is the fundamental process leading to a suppression of quantum mechanical interference and therefore is indispensable for our understanding of the appearance of the classical world. The destruction of phase coherence in a quantum system occurs due to interactions with its environment and can be studied, e.g., in mesoscopic metals and semiconductors where the quantum-mechanical wave nature of the electrons leads to a variety of transport phenomena at low temperatures.

Although the concrete definition of the dephasing rate, $1 / \tau_{\varphi}$, depends on the experiment used to determine it, the electron-electron interactions are thought to be the dominant mechanism for the destruction of phase coherence in metals without dynamical impurities below about $1 \mathrm{~K}$. The dephasing rate for interacting electrons in a diffusive environment was first calculated by Altshuler, Aronov, and Khmelnitsky (AAK) and vanishes at low temperatures, $T$, with some power of $T$, depending on the dimensionality of the system. ${ }^{1}$

In the last decade several independent groups performed magnetoresistance experiments $\mathrm{s}^{2-5}$ to probe the influence of dephasing on weak localization in disordered metallic wires. Irritatingly, a saturation of the dephasing rate, $1 / \tau_{\varphi}$, has been observed at the lowest experimentally accessible temperatures. This observation has triggered an intense discussion on the mechanism responsible for the excess of dephasing. ${ }^{6-8}$ The most promising candidates to explain the saturation of $1 / \tau_{\varphi}$ are extremely low concentrations of dynamical impurities, such as atomic two-level systems ${ }^{9,10}$ or magnetic impurities. ${ }^{3-5,11-14}$ This has been corroborated on the one hand by experiments ${ }^{3,5}$ on extremely clean $\mathrm{Ag}$ and $\mathrm{Au}$ samples where the dephasing rate continues to decrease well below $100 \mathrm{mK}$ and on the other hand by doping studies with magnetic impurities. ${ }^{2,3,5,15,16}$ As expected theoretically, ${ }^{12}$ these experiments show a saturation of $1 / \tau_{\varphi}$ above the Kondo temperature, $T_{\mathrm{K}}$, the characteristic scale of screening of the magnetic moment, and a suppression of $1 / \tau_{\varphi}$ below this scale. Recent highly controlled experiments, ${ }^{15,16}$ in which a few ppm (parts per million) of Fe ions have been implanted by ion beam lithography into very clean $\mathrm{Ag}$ samples, showed that the screening of these Fe ions is surprisingly well described ${ }^{15}$ by the theoretically predicted dephasing rate for spin-1/2 Kondo impurities ${ }^{13}$ down to temperatures of $0.1 T_{\mathrm{K}}$. At the lowest temperature, again a plateau in the dephasing rate has been observed proportional to the number of implanted $\mathrm{Fe}$ ions. The origin of this puzzling behavior is still unclear but may arise from further dynamical defects created during the implantation process or by rare $\mathrm{Fe}$ ions with a different chemical environment and strongly reduced magnetic screening.

An obvious option to study the influence of magnetic impurities on the dephasing rate is to measure its dependence on an externally applied magnetic field. The application of sufficiently large magnetic fields freezes out inelastic spinflip processes as discussed theoretically in Ref. 12, and therefore one expects the dephasing rate to return to the value predicted by AAK for dephasing induced by Coulomb interactions in a diffusive environment. The orbital contribution of the magnetic field does, however, destroy the weaklocalization (WL) contribution to the magnetoresistance, as the joint propagation of an electron and a hole along timereversed trajectories (the Cooperon) picks up extra (random) Aharonov-Bohm phases in the presence of external magnetic fields. Measuring the $B$-dependent dephasing rate in a WL experiment is therefore only possible in strictly one- or twodimensional systems using magnetic fields almost exactly parallel to such a structure, requiring an accurate alignment of magnetic fields.

Universal conductance fluctuations (UCF) and AharonovBohm (AB) oscillations with a periodicity of $h / e$, on the other hand, are not suppressed by orbital effects and can be used rather directly to determine the field dependence of the dephasing rate. ${ }^{17}$

UCFs can be observed as characteristic fluctuations of the conductance as a function of the magnetic field. The external magnetic field enters the metal and changes the pattern of the electrons wave functions and therefore the conductance in a random but reproducible way ("magnetofingerprint"). In $A B$ experiments performed on mesoscopic rings, these sample fluctuations are further modulated by periodic $h / e$ oscillations resulting from the magnetic flux piercing the ring. Both $\mathrm{UCF}$ and $\mathrm{AB}$ oscillations rely on the constructive interference occurring in the collective propagation of electrons and holes traveling along the same path (the diffuson). These are robust against the breaking of time-reversal invariance (while Cooperon contributions are rapidly suppressed by 
small fields) but are sensitive to dephasing by inelastic processes. Indeed, Benoit et al. ${ }^{18}$ and Pierre et al. ${ }^{5}$ have shown that the amplitude of Aharonov-Bohm oscillations increases by almost an order of magnitude for increasing magnetic fields, clearly showing a suppression of dephasing by magnetic (Zeeman) fields. This leads to the conclusion that the main mechanism of dephasing in the investigated lowtemperature regime is the scattering from magnetic impurities.

Previously we have studied the zero-field dephasing rate due to diluted Kondo impurities as measured from the WL experiment. ${ }^{13}$ We showed that the dephasing rate for all experimentally relevant temperatures is proportional to the inelastic cross section, ${ }^{14}$ which itself can be expressed in terms of the $T$ matrix describing the scattering of the electrons from a single magnetic impurity. Such a relation has been proposed previously by Schwab and Eckern ${ }^{19}$ in the context of UCFs.

In this paper we generalize our previous work to arbitrary, diluted impurity scatterers with typical energy transfer larger than $1 / \tau_{\varphi}$. Furthermore, we supply the magnetic field dependence of $1 / \tau_{\varphi}$ as measured in the $\mathrm{AB}$ experiment (and compare our results to the $\mathrm{AB}$ experiments performed by Pierre and Birge ${ }^{20}$ ). The outline of this paper is as follows: In Sec. II we discuss the dephasing rate due to generic dynamical scatterers as measured in the WL experiment. We briefly review our previous results ${ }^{13}$ and generalize them to arbitrary dynamical impurities with typical energy transfer larger than $1 / \tau_{\varphi}$. In Sec. III we turn to the dephasing rate as measured from the UCF and the amplitude of the AB oscillations. We briefly review the main concepts entering the analysis of these experiments and discuss how the dephasing rate measured from the UCF differs from that measured in the WL experiment. The main goal of Sec. III is to give the dephasing rate as measured from the amplitude of the $\mathrm{AB}$ oscillations. Results for the dephasing rate obtained using the numerical renormalization group (NRG) are described in Sec. IV. Section V summarizes with a discussion.

\section{DEPHASING RATE FROM WEAK-LOCALIZATION CORRECTIONS TO THE CONDUCTIVITY}

The most accurate way to extract $1 / \tau_{\varphi}$ at low magnetic fields is via the WL corrections to the Drude conductivity, which result from coherent back scattering of an electronhole pair traveling along time-reversed paths in the disordered environment. Technically, the coherent propagation of the electron-hole pair is described by the Cooperon, $C_{\Omega}(\mathbf{q})$, and the WL correction is given by

$$
\Delta \sigma_{\mathrm{WL}}^{0}=-\frac{2 e^{2} D}{\pi} \int \frac{d^{d} \mathbf{q}}{(2 \pi)^{d}} C_{\Omega=0}(\mathbf{q}),
$$

see Fig. 1. In the absence of dephasing by inelastic processes $C_{\Omega}(\mathbf{q})$ is the bare Cooperon,

$$
C_{\Omega}^{0}(\mathbf{q})=\frac{1}{D \mathbf{q}^{2}+i \Omega+1 / \tau_{B}},
$$

as diagrammatically depicted in Fig. 2 and $^{21}$

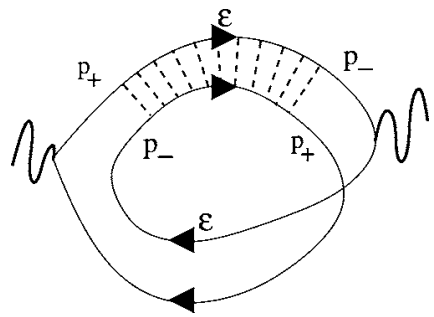

FIG. 1. Diagrammatic representation of the Cooperon $C_{\Omega=0}^{0}(q)$ which enters the WL corrections to the Drude conductivity. $p_{ \pm}=q / 2 \pm p$ and wavy lines denote current operators.

$$
\begin{gathered}
\frac{1}{\tau_{B}}=4 D e B, \quad d=2 \quad(\mathbf{n} \| \mathbf{B}), \\
\frac{1}{\tau_{B}}=\frac{D}{3}\left(e B L_{\perp}\right)^{2}, \quad d=1,2 \quad(\mathbf{n} \perp \mathbf{B}),
\end{gathered}
$$

is the dephasing rate due to the applied magnetic field, $B$. Here $D$ is the diffusion constant, $d$ is the dimension of the diffusion process, $\tau$ denotes the mean scattering time corresponding to a mean free path $l=v_{\mathrm{F}} \tau, \mathbf{n}$ is a unit vector orthogonal to the probe in $d=2$, and pointing along the wire in $d=1$ and $L_{\perp}$ is the transverse dimension of the sample. As can be seen from Eq. (1) the WL corrections depend on the strength of the applied magnetic field, $B$, and diverge in low dimensions, $d=1,2$ for $B=0$, reflecting the fact that WL corrections in low-dimensional systems may become strong and lead to strong Anderson localization.

Taking into account interactions (as, e.g., provided by dynamical impurities) the bare Cooperon dresses with a mass, $1 / \tau_{\varphi}$, i.e. (if purely exponential decay is guaranteed),

$$
C_{\Omega=0}(\mathbf{q})=\frac{1}{D \mathbf{q}^{2}+1 / \tau_{\varphi}+1 / \tau_{B}}
$$

For weak magnetic fields $\left(\tau_{\varphi} \ll \tau_{B}\right)$ the WL corrections are therefore cut off by $1 / \tau_{\varphi}$, allowing to determine the dephasing rate from fitting the magnetoresistance.

In this section we study the dephasing rate due to generic diluted, dynamical scatterers with typical energy transfer larger than $1 / \tau_{\varphi}$ as measured from WL. To be specific, we consider a Hamiltonian of the general form

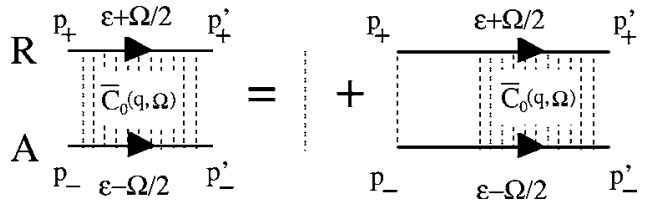

FIG. 2. Bethe-Salpeter equation for the bare Cooperon $\bar{C}_{0}(q, \Omega)=\left(1 / 2 \pi \nu \tau^{2}\right) C_{0}(q, \Omega), \quad p_{ \pm}=q / 2 \pm p, \quad$ and $\quad p_{ \pm}^{\prime}=q / 2 \pm p^{\prime}$. Dashed lines denote scattering from static impurities and $R, A$ denotes the particle and hole lines, respectively. 


$$
H_{\mathrm{imp}}=H_{\mathrm{imp}}^{0}+\sum_{i} c_{\mathbf{k} \sigma^{\dagger}}^{\dagger} c_{\mathbf{k}^{\prime} \sigma^{\prime}} f_{\mathbf{k k}^{\prime} \sigma \sigma^{\prime}}^{\alpha} \hat{X}_{\alpha} e^{i\left(\mathbf{k}-\mathbf{k}^{\prime}\right) \mathbf{x}_{i}}
$$

where $H_{\mathrm{imp}}^{0}$ is the Hamiltonian of the isolated impurity, $c^{\dagger}, c$ are creation and annihilation operators of conduction band electrons, $\mathbf{x}_{i}$ denotes the position of the impurities, and the momentum and spin-dependent function $f^{\alpha}$ parametrizes the coupling to some operator $\hat{X}_{\alpha}$ describing transitions of the internal states of the dynamical impurity. Equation (6), e.g., describes the coupling of the conduction band electrons to Kondo impurities, two-level systems, etc. In order to find the dephasing rate due to such generic impurities one has to compute the "self energy" or "mass" of the Cooperon generated by the operators of Eq. (6).

Two assumptions allow to reduce this problem to that of summing up a simple geometric series. First, we assume that the concentration of dynamical impurities $n_{i}$ is small, and, second, that the elastic mean free path $l$ is large. How small $n_{i}$ and how large $l$ has to be, depends on both, the dynamics and the extension of the impurity as briefly discussed below and-for Kondo impurities-in more detail in Ref. 13 (the influence of stronger disorder on the distribution of Kondo temperatures has recently been investigated by Kettemann and Mucciolo $^{22}$ ). Note that in relevant experimental systems in the WL regime ${ }^{2-5}$ the two assumptions are well justified. ${ }^{13}$ The first observation is that quantum interference corrections to the inelastic scattering rate are small when a diffusing electron is unlikely to return to the same dynamical impurity (i.e., when the weak localization corrections are weak). Technically speaking, this is reflected in the fact that diagrams mixing scattering from dynamical and static impurities are suppressed by factors of $1 / N_{\perp}$ and $a / l$ [or $1 /\left(k_{F} l\right)$ for $\left.a<1 / k_{F}\right]$, where $N_{\perp}$ is the number of transverse channels in a quasi one or two dimensional system and $a$ is the typical diameter of the dynamical impurity. This effect and further system dependent factors relevant for the suppression of quantum interference corrections are discussed in Ref. 13. Only at lowest, experimentally unprobed temperatures, does the enhanced infrared singularity, caused by the presence of extra diffusion modes, overcompensate this phase space suppression factor, as discussed in detail in Ref. 13. The small parameter $1 /\left(k_{\mathrm{F}} l\right)$ or $a / l$ therefore reduces the problem to compute the "mass" of the Cooperon to that of solving the Bethe-Salpeter equation diagrammatically depicted in Fig. 3(a).

For small $n_{i}$, one can furthermore restrict the analysis of the irreducible vertex $\Gamma$ to terms linear in $n_{i}$ as shown in Fig. 3(b). $\Gamma$ can be separated into three distinct contributions: self-energy diagrams [the first two terms in Fig. 3(b)], an "elastic" vertex correction with no energy transfer between upper and lower line (third term), and an "inelastic" vertex where interaction lines connect the two lines (last term). Only this inelastic vertex makes the Bethe-Salpeter equation a true integral equation as it mixes frequencies but, fortunately, this term can be neglected ${ }^{1}$ if the typical energy, $\Delta E$, exchanged between electrons and holes during an interaction process greatly exceeds the dephasing rate due to the dynamical impurities, $1 / \tau_{\varphi}$, i.e., $\Delta E \tau_{\varphi} \gg 1$. Physically, ${ }^{1}$ the suppression of the inelastic vertex arises as an exchange of en-

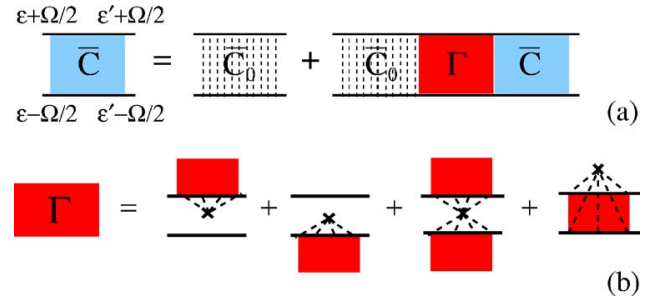

FIG. 3. (Color online) Bethe-Salpeter equation for the Cooperon $\bar{C}$ in the presence of dilute dynamical impurities to linear order in $n_{i} . \bar{C}_{0}$ is the bare Cooperon in the absence of interactions and $\Gamma$ the irreducible vertex obtained by adding self-energy, elastic-, and inelastic-vertex contributions. The crosses with attached dashed lines denote the averaging over impurity positions $\mathbf{x}_{i}$, the squares the inelastic scattering from a single impurity to arbitrary order.

ergy $\Delta E$ leads to a phase mismatch of order $e^{i \Delta E \tau_{\varphi}}$ between electron and hole destroying interference completely for $\Delta E \tau_{\varphi} \gg 1$. Technically, one can confirm this argument by estimating corrections to the WL contributions due to the inelastic vertex, as, e.g., depicted in Fig. $4 .{ }^{13}$ More importantly, however, the condition $\Delta E \tau_{\varphi} \gg 1$ always holds for sufficiently small concentrations $n_{i}$, since $1 / \tau_{\varphi} \propto n_{i}$. In the case of Kondo impurities discussed below, for example, this condition translates to $n_{i} \ll \nu T_{\mathrm{K}}$.

Restricting to the self-energy and elastic vertex contributions, the Bethe-Salpeter equation is easily solved: Since self-energy and elastic vertex contributions conserve the energy of single electron lines, the solution of the reduced Bethe-Salpeter equation amounts to a straightforward summation of a geometric series. Setting the center-of-mass frequency $\Omega$ to 0 (see Fig. 1 and Fig. 3), the Cooperon is given by

$$
C_{\Omega=0}(\epsilon, \mathbf{q})=\frac{1}{D \mathbf{q}^{2}+1 / \tau_{\varphi}(\epsilon, T)+1 / \tau_{B}},
$$

with the $T$ and $\epsilon$ dependent dephasing rate

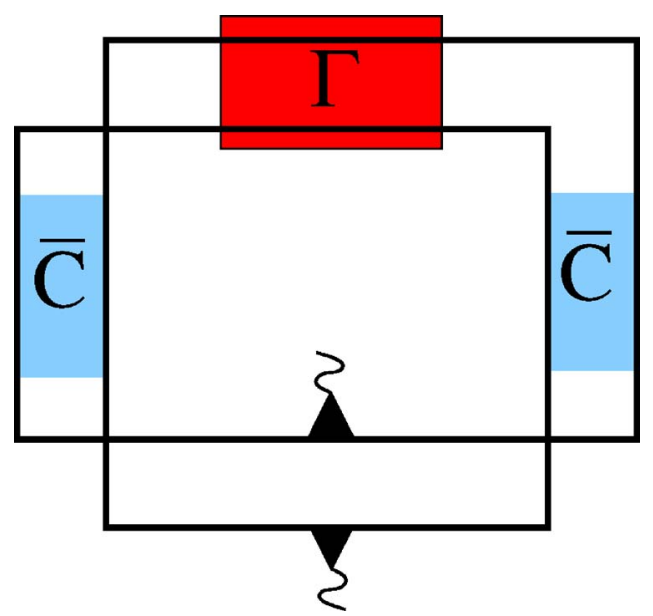

FIG. 4. (Color online) Lowest order correction to WL contributions due to inelastic vertex. The Cooperon, $\bar{C}$, is dressed with a mass resulting from summation of the elastic part of the vertex $\Gamma$, i.e., the self-energy and the elastic vertex contribution. 


$$
\begin{aligned}
\frac{1}{\tau_{\varphi}(\epsilon, T)}= & \frac{2 n_{i}}{\pi \nu}\left[\int \frac{d^{3} \mathbf{p}}{(2 \pi)^{3}} g_{\epsilon}(\mathbf{p}) \frac{1}{2 i}\left[T_{-\mathbf{p},-\mathbf{p}}^{\mathrm{A}}(\epsilon)-T_{\mathbf{p p}}^{\mathrm{R}}(\epsilon)\right]\right. \\
& \left.-\int \frac{d^{3} \mathbf{p}}{(2 \pi)^{3}} \int \frac{d^{3} \mathbf{p}^{\prime}}{(2 \pi)^{3}} g_{\epsilon}(\mathbf{p}) g_{\epsilon}\left(\mathbf{p}^{\prime}\right) T_{\mathbf{p} \mathbf{p}^{\prime}}^{\mathrm{R}}(\epsilon) T_{-\mathbf{p},-\mathbf{p}^{\prime}}^{\mathrm{A}}(\epsilon)\right] .
\end{aligned}
$$

Here $g_{\epsilon}(\mathbf{p})=\frac{\pi / 2 \tau}{[\epsilon(\mathbf{p})-\epsilon]^{2}+1 / 4 \tau^{2}}$ restricts the electrons momenta, $\mathbf{p}$, and energies, $\epsilon$, to the Fermi-surface, $\epsilon(\mathbf{p})$ is the dispersion relation of the conduction band, $T^{\mathrm{A}, \mathrm{R}}$ are the advanced and/or retarded $T$ matrices, defined by the Green's function $G_{\mathbf{x x}^{\prime}}(\epsilon)=G_{\mathbf{x x}^{\prime}}^{0}(\epsilon)+G_{\mathbf{x} \mathbf{0}}^{0}(\epsilon) T(\epsilon) G_{\mathbf{0 x}}^{0}{ }^{\prime}(\epsilon)$, and $\nu$ denotes the density of states per spin. Equation (8) generalizes our result of Ref. 13 to arbitrary shaped diluted impurities. Notice that also forward scattering processes enter $1 / \tau_{\varphi}$, which do not contribute to the transport scattering rate. We stress that Eq. (8) is the general result for the dephasing rate for a weakly disordered metal due to a low concentration of generic dynamical impurities for which the condition $\Delta E \gg 1 / \tau_{\varphi}$ holds. In the opposite limit, $\Delta E \gg 1 / \tau_{\varphi}$, vertex corrections become important, as has been discussed earlier on ${ }^{23,24}$ in the context of magnetic impurities.

As we assumed that $a \ll l$, Eq. (8) can be further simplified,

$$
\begin{aligned}
\frac{1}{\tau_{\varphi}(\epsilon, T)}= & \frac{2 n_{i}}{\pi \nu}\left[\int_{S_{F}^{\epsilon}} \frac{d^{2} \mathbf{p}}{(2 \pi)^{3}} \frac{1}{\left|v_{\mathrm{F}}(\mathbf{p})\right|} \operatorname{Im}\left[\pi T_{\mathbf{p p}}^{\mathrm{A}}(\boldsymbol{\epsilon})\right]\right. \\
& \left.-\int_{S_{F}^{\epsilon}} \frac{d^{2} \mathbf{p}}{(2 \pi)^{3}} \int_{S_{F}^{\epsilon}} \frac{d^{2} \mathbf{p}^{\prime}}{(2 \pi)^{3}} \frac{1}{\left|v_{\mathrm{F}}(\mathbf{p})\right|} \frac{1}{\left|v_{\mathrm{F}}\left(\mathbf{p}^{\prime}\right)\right|}\left|\pi T_{\mathbf{p p}^{\prime}}^{\mathrm{R}}(\epsilon)\right|^{2}\right],
\end{aligned}
$$

where $S_{F}^{\epsilon}$ is the Fermi surface (or more precisely the surface with $\epsilon_{k}=\epsilon$ ). Here we also assumed a time-reversal invariant system with $T_{\mathbf{p p}^{\prime}}^{\mathrm{R}}(\epsilon)=T_{-\mathbf{p}^{\prime},-\mathbf{p}}^{\mathrm{R}}(\boldsymbol{\epsilon})$ and employed the identity $\left[T_{\mathbf{p p}^{\prime}}^{\mathrm{R}}(\epsilon)\right]^{*}=T_{\mathbf{p}^{\prime} \mathbf{p}}^{\mathrm{A}}(\epsilon)$. Also Kettemann and Mucciolo have generalized the dephasing rate for a momentum independent $T$ matrix to Eq. (9) independently in a recent report. ${ }^{22}$ The dephasing rate given in Eq. (9) has a simple interpretation: ${ }^{14}$ Since the Fermi-surface integrated imaginary part of the $T$ matrix is proportional to the total cross section and $\left|T_{\mathbf{p p}^{\prime}}^{\mathrm{R}}\right|^{2}$ (integrated over the Fermi surface) is proportional to the elastic cross section, its difference is, by definition, proportional to the inelastic cross section, $\sigma_{\text {inel }}$, introduced in Ref. 14. Therefore, Eq. (9) can be rewritten in the form

$$
\frac{1}{\tau_{\varphi}(\boldsymbol{\epsilon})}=n_{i}\left\langle v_{F}(\mathbf{p}) \sigma_{\text {inel }}(\mathbf{p}, \boldsymbol{\epsilon})\right\rangle
$$

where $\langle\cdots\rangle$ denotes an angular average weighted by $1 / v_{F}(\mathbf{p})$ to take into account that fast electrons are scattered more frequently from elastic impurities. According to Eq. (10), $\tau_{\varphi}$ is nothing but the average time needed (in a semiclassical picture) to scatter from an impurity with cross section $\sigma_{\text {inel }}$. Note that the vanishing of $1 / \tau_{\varphi}$ for static impurities is guaranteed by the optical theorem.

From Eq. (9) we can read off the dephasing rate for diluted dynamical isotropic $s$-wave scatterers ${ }^{13,19}$

$$
\frac{1}{\tau_{\varphi}(\epsilon, T)}=\frac{2 n_{\mathrm{i}}}{\pi \nu}\left[\pi \nu^{\operatorname{loc}} \operatorname{Im}\left[T^{\mathrm{A}}(\epsilon)\right]-\left|\pi \nu^{\mathrm{loc}} T^{\mathrm{R}}(\epsilon)\right|^{2}\right],
$$

where $\nu^{\text {loc }}$ is the local density of states at the Fermi energy at the site of the impurity which can differ from the thermodynamic density of states entering the prefactor. Note that in the case of Kondo impurities discussed below (and in Ref. 13), the combination $\nu^{\text {loc }} T^{R / A}(\epsilon)=f\left(\epsilon / T_{\mathrm{K}}, T / T_{\mathrm{K}}, B / T_{\mathrm{K}}\right)$ is an universal dimensionless function of the ratios $\epsilon / T_{\mathrm{K}}, T / T_{\mathrm{K}}, B / T_{\mathrm{K}}$. If the assumptions underlying the derivation of Eq. (11) are valid, one can therefore predict without any free parameter the dephasing rate if the concentration of spin-1/2 impurities, the Kondo temperature and the thermodynamic density of states are known (see, e.g., Ref. 15). However, one of the assumptions underlying the derivation of the prefactor of Eq. (11) may not be valid in realistic materials: we assumed that the static impurities are completely uncorrelated and local such that electrons are scattered uniformly over the Fermi surface. While this should be a good assumption in doped semiconductors, this may not be valid in metals with complex Fermi surfaces and strongly varying Fermi velocities. Under the latter conditions, we expect that the prefactor of Eq. (11) becomes nonuniversal, yielding temperature-independent corrections of order one, which may be important for the interpretation of highprecision experiments. ${ }^{16,15}$

In Ref. 13 we have calculated the leading corrections to Eq. (11) arising from mixed diagrams involving combined scattering from static and dynamical impurities and from diagrams including higher processes in $n_{i}$. We showed that, suppressed by the small parameter $1 /\left(k_{\mathrm{F}} l\right)$, their contributions are negligible at all experimentally relevant temperatures, $T$. Only at the lowest experimentally irrelevant temperatures do these corrections become important due to infrared singularities of the dressed interaction potential (dressed by coherent backscattering processes). The estimates of subleading corrections presented in Ref. 13 can be generalized to extended dynamical impurities by replacing $1 /\left(k_{F} l\right)$ by $a / l$ for $k_{F} a>1$.

The experimentally measured dephasing rate does not resolve the dependence on the electrons energy $\epsilon$. We described in Ref. 13 that to allow for a comparison with the $\epsilon$-independent dephasing rate, $\tau_{\varphi}^{-1}(T)$, extracted from the WL experiment, the energy-resolved representations of $1 / \tau_{\varphi}$, Eqs. (9)-(11), still require an average over energies according to

$$
\frac{1}{\tau_{\varphi}(T)}=\left\{\begin{array}{l}
{\left[-\int d \epsilon f_{\mathrm{F}}^{\prime}(\epsilon) \tau_{\varphi}(\epsilon, T)^{(2-d) / 2}\right]^{2 /(d-2)}, \quad d=1,3,} \\
\frac{1}{\tau} \exp \left[\int d \epsilon f_{\mathrm{F}}^{\prime}(\epsilon) \ln \frac{\tau_{\varphi}(\epsilon, T)}{\tau}\right], \quad d=2, \\
-\int d \epsilon f_{\mathrm{F}}^{\prime}(\epsilon) / \tau_{\varphi}(\epsilon, T), \quad \tau_{\varphi} / \tau_{B} \gg 1 .
\end{array}\right.
$$

Here the last line applies to a case where a relatively strong magnetic field, $B$, is present. 
Specifying to a situation where the coupling of the conduction band electrons to the (diluted) dynamical impurities is described by the spin-1/2 Kondo effect, the general Hamiltonian of Eq. (6) takes the form

$$
H_{\mathrm{S}}=J \sum_{i} \hat{\mathbf{S}}_{i} c_{\sigma}^{\dagger}\left(\mathbf{x}_{i}\right) \sigma_{\sigma \sigma^{\prime}} c_{\sigma^{\prime}}\left(\mathbf{x}_{i}\right),
$$

where $J$ is the exchange constant. An external magnetic field, $B$, causes Zeeman splitting, $\epsilon_{z}$, of the conduction band electron spin states,

$$
\epsilon_{z}=g_{e} \mu_{B} B
$$

and couples to the impurity spins according to

$$
H_{B}=g_{S} \mu_{B} B \sum_{i} \hat{\mathbf{S}}_{i}^{z}
$$

$g_{e}, g_{S}$ are the electrons and the magnetic impurities gyromagnetic factors, respectively. As already mentioned in the Introduction, measuring the $B$-field dependence of the dephasing rate due to Kondo impurities in a WL experiment is a highly delicate task. In view of this difficulty it is more feasible to measure the $B$ dependence of $1 / \tau_{\varphi}$ from the amplitude of the $\mathrm{AB}$ oscillations as discussed in the following section.

\section{DEPHASING RATE FROM UNIVERSAL CONDUCTANCE FLUCTUATIONS AND AHARANOV- BOHM OSCILLATIONS}

Let us first begin with a brief discussion of the universal conductance fluctuations (UCF) and their dependence on $1 / \tau_{\varphi}$ and then turn to the experiment on Aharonov-Bohm rings.

To be specific, we consider a wire of noninteracting electrons, scattering elastically from static impurities, and inelastically off a low concentration, $n_{i}$, of Kondo impurities, where the coupling of the conduction band electrons to the dynamical impurities is described by the Hamiltonian equation (13) and the influence of the magnetic field is accounted for by Eqs. (14) and (15).

For wires of length $L \gg L_{T}=\sqrt{D / T}$, the fluctuations of the conductance, $\overline{\delta g \delta g}$, are determined ${ }^{25}$ by

$$
\begin{aligned}
\overline{\delta g \delta g}= & \frac{\left(2 e^{2} D\right)^{2}}{3 \pi T L^{4}} \int d \epsilon_{1} d \epsilon_{2} f_{\mathrm{F}}^{\prime}\left(\epsilon_{1}\right) f_{\mathrm{F}}^{\prime}\left(\epsilon_{2}\right) \\
& \times \int d \mathbf{x}_{1} d \mathbf{x}_{2}\left|P_{\epsilon_{1}, \epsilon_{2}}\left(\mathbf{x}_{1}, \mathbf{x}_{2}\right)\right|^{2} .
\end{aligned}
$$

Here $P_{\epsilon_{1}, \epsilon_{2}}\left(\mathbf{x}_{1}, \mathbf{x}_{2}\right)$ is the amplitude for an electron-hole pair, with energies $\epsilon_{1}, \epsilon_{2}$, respectively, to diffusively travel from $\mathbf{x}_{1}$ to $\mathbf{x}_{2}$ along the same trajectory (diffuson, see Fig. 5). The overbar denotes the ensemble average, which is experimentally realized by changing the magnetic field. Equation (16) assumes the large ring diameters, $L \gg L_{\varphi}$, such that the dephasing rate, $1 / \tau_{\varphi}$, controls the magnitude of the fluctuations. Furthermore, we assume temperatures $T \tau_{\varphi} \gg 1$. Notice that generally there is also a contribution from the Cooperon, which is, however, suppressed already for small magnetic fields.

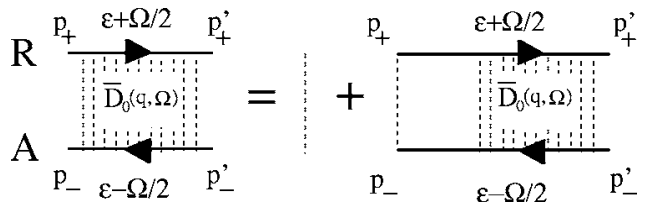

FIG. 5. Bethe-Salpeter equation for the bare diffuson $\bar{D}_{0}(q, \Omega)$ $=\left(1 / 2 \pi \nu \tau^{2}\right) D_{0}(q, \Omega) . p_{ \pm}=p \pm q / 2$ and $p_{ \pm}^{\prime}=p^{\prime} \pm q / 2$. Dashed lines denote scattering from static impurities and $R, A$ denotes the particle and hole lines, respectively.

We change to momentum representation and separate the two-particle propagator $P$ into its spin-singlet and -triplet components, $P=\sum_{i=1, \ldots, 4} P^{(i)}$, where (following the notation of Ref. 12)

$$
P_{\epsilon_{1} \epsilon_{2}}^{(i)}(\mathbf{q})=\frac{1}{D \mathbf{q}^{2}+i\left(\epsilon_{1}-\epsilon_{2}+\zeta_{i} \epsilon_{z}\right)+1 / \tau_{S O}^{(i)}+1 / \tau_{\varphi, S}^{(i)}\left(\epsilon_{1}, \epsilon_{2}, B\right)} .
$$

The modes $i=1,2,3$ describe the spin triplet state with $S_{z}$ component equal to $1,-1$, and 0 , respectively. $i=4$ denotes the spin singlet channel. The Zeeman splitting enters only the triplet diffuson with nonvanishing projection $S_{z}= \pm 1$, i.e., $\zeta_{i}= \pm 1$ for $i=1,2$ and zero otherwise. $1 / \tau_{\text {SO }}^{(i)}$ is the spin-orbit scattering rate. $1 / \tau_{\text {SO }}^{(i)}$ is identical for the three spin triplet diffuson $(i=1,2,3)$ and zero for the spin singlet mode $(i=4)$. For strong spin-orbit scattering only the singlet diffusion contributes (otherwise $1 / \tau_{\mathrm{SO}}^{(i)}$ is an additional fitting parameter). Finally $1 / \tau_{\varphi, S}^{(i)}$ is the dephasing rate for the $i$ th diffuson mode due to the presence of diluted magnetic impurities which has the structure

$$
\begin{aligned}
\frac{1}{\tau_{\varphi, S}^{(i)}\left(\epsilon_{1}, \epsilon_{2}\right)}= & \frac{2 n_{i}}{\pi \nu}\left(\frac{\pi \nu}{2 i}\left[\mathrm{~T}^{(i, a)}\left(\epsilon_{2}, B\right)-\mathrm{T}^{(i, b)}\left(\epsilon_{1}, B\right)\right]\right. \\
& \left.-(\pi \nu)^{2} \mathrm{~T}^{(i, c)}\left(\epsilon_{1}, B\right) \mathrm{T}^{(i, d)}\left(\epsilon_{2}, B\right)\right),
\end{aligned}
$$

where the proper combination of $T$ matrices for the various channels can be read off by comparison with Table I. Equation (18) is evaluated from summing up self-energy and elastic vertex contributions. Notice that in contrast to the WL experiment the electron and hole lines (i.e., the inner and outer rings) in Fig. 6 represent different measurements. Therefore there are no correlations between dynamical im-

TABLE I. Combination of $T$ matrices entering the dephasing rates for spin-triplet and spin-singlet diffusons. $S$ denotes the total spin and $M$ its $z$ component. $T_{\uparrow}, T_{\downarrow}$ denotes the $T$ matrix for spin-up and spin-down electrons, respectively.

\begin{tabular}{lcc}
\hline \hline$i$ & $|S, M\rangle$ & Combinations of $T$ matrices \\
\hline 1 & $S=1, M=1$ & $T^{1}=\frac{1}{2 i}\left(T_{\downarrow}^{\mathrm{A}}-T_{\uparrow}^{\mathrm{R}}\right)-T_{\downarrow}^{\mathrm{R}} T_{\uparrow}^{\mathrm{A}}$ \\
2 & $S=1, M=-1$ & $T^{2}=\frac{1}{2 i}\left(T_{\uparrow}^{\mathrm{A}}-T_{\downarrow}^{\mathrm{R}}\right)-T_{\uparrow}^{\mathrm{R}} T_{\downarrow}^{\mathrm{A}}$ \\
3 & $S=1, M=0$ & $T^{3}=\frac{1}{2} \operatorname{Im}\left(T_{\uparrow}^{\mathrm{A}}+T_{\downarrow}^{\mathrm{A}}\right)-\frac{1}{2}\left(T_{\uparrow}^{\mathrm{R}} T_{\uparrow}^{\mathrm{A}}-T_{\downarrow}^{\mathrm{R}} T_{\downarrow}^{\mathrm{A}}\right)$ \\
4 & $S=0$ & $T^{4}=\frac{1}{2} \operatorname{Im}\left(T_{\uparrow}^{\mathrm{A}}+T_{\downarrow}^{\mathrm{A}}\right)-\frac{1}{2}\left(T_{\uparrow}^{\mathrm{R}} T_{\uparrow}^{\mathrm{A}}-T_{\downarrow}^{\mathrm{R}} T_{\downarrow}^{\mathrm{A}}\right)$ \\
\hline \hline
\end{tabular}




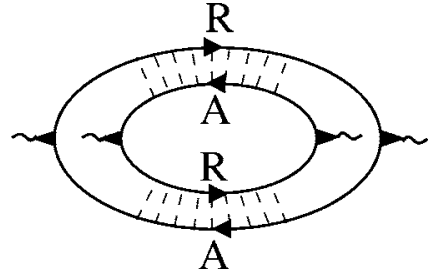

FIG. 6. Diagram giving the main contribution to the UCF. Dashed lines represent coherent impurity scattering of electron $(R)$ hole $(A)$ pair, i.e., the bare diffuson where interaction due to scattering from magnetic impurities is not yet taken into account.

purities residing on different rings and interaction lines may only be drawn within the same ring. Consequently the inelastic vertex contributions do not enter the Bethe-Salpeter equation for the diffuson, see Fig. 7. Notice that there are inelastic vertex contributions, as, e.g., depicted in Fig. 8, which become important in the context of electron-electron interactions. ${ }^{26}$ It is instructive to compare those to the inelastic vertex corrections relevant for WL depicted in Fig. 4. In the latter case, the sum of the incoming momenta of the vertex is small due to the Cooperon in Fig. 4. Consequently, the inelastic vertex corrections to the WL dephasing rate are not suppressed by powers of $1 /\left(k_{\mathrm{F}} l\right)$ but only by powers of $1 /\left(\Delta E \tau_{\varphi}\right)$. In contrast, the relevant momenta in Fig. 8 are uncorrelated (i.e., particle and hole are far apart), leading to an suppression both by powers of $1 /\left(k_{\mathrm{F}} l\right)$ and of $1 /\left(\Delta E \tau_{\varphi}\right){ }^{27}$

We point out the following differences for $1 / \tau_{\varphi, S}$ measured from the UCF experiment, Eq. (18), compared to that found from the WL, Eq. (11). First, the $T$ matrices entering Eq. (18) depend on the spin configuration of the diffuson mode and have acquired a $B$ dependence due to the coupling of the impurity spin to $B$, Eq. (15). Second, $1 / \tau_{\varphi, S}$ depends on two energies. This results from the fact that in the UCF experiment electron and hole lines constituting the diffuson are produced in different measurements of the conductance (see Fig. 6). Therefore their energies are individually averaged as can be seen in Eq. (16).

From Eqs. (16) and (17) the amplitude of the UCFs is obtained to be proportional to $\sqrt{\tau_{\varphi, S}}$. Especially compared to the $\mathrm{AB}$ oscillations, discussed below, the $1 / \tau_{\varphi, S}$ dependence of the UCFs is rather weak. In the following, we will there-

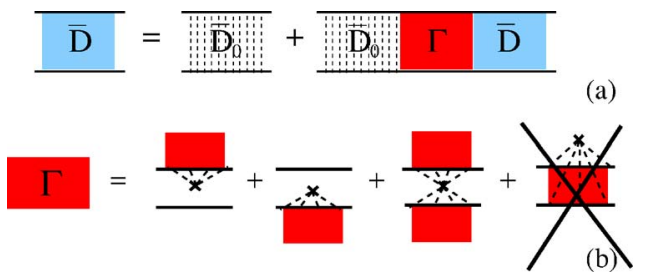

FIG. 7. (Color online) (a) Bethe-Salpeter equation for the diffuson, $\bar{D}$, in the presence of (dilute) magnetic impurities to linear order in $n_{\mathrm{i}} . \bar{D}_{0}$ is the bare diffuson in the absence of interactions. (b) Diagrammatic representation of the irreducible interaction vertex, $\Gamma$, consisting of the self-energy (represented by the first two contributions), the elastic vertex (third contribution). The inelastic vertex (the fourth contribution) does not enter the diffuson as measured in the UCF.

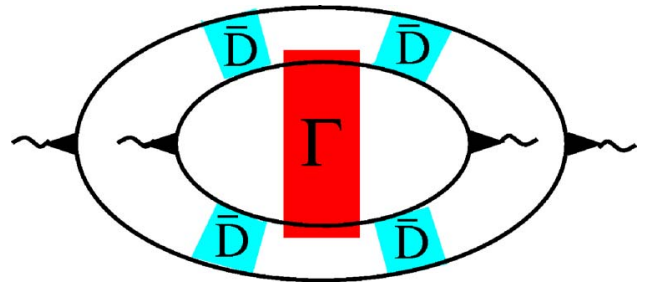

FIG. 8. (Color online) Diagrammatic representation of lowestorder corrections to UCF due to inelastic vertex contributions. Notice that for a local interaction these are not only suppressed by powers of $1 /\left(\Delta E \tau_{\varphi}\right)$ but also small in powers $1 /\left(k_{\mathrm{F}} l\right)$.

fore focus our discussion on $\mathrm{AB}$ experiments.

Aharonov-Bohm oscillations are measured in a ring geometry, ${ }^{20,28}$ where the conductance oscillates periodically as a function of $B$ piercing the ring, and the amplitude of these $\mathrm{AB}$ oscillations decrease exponentially with $1 / \tau_{\varphi, S}$. The periodic oscillations result from the change of boundary conditions, due to flux lines piercing the ring and can be calculated from

$$
\begin{aligned}
\overline{\delta g(\phi) \delta g(\phi+\Delta \phi)}= & \frac{\left(2 e^{2} D\right)^{2}}{3 \pi T L^{4}} \int d \epsilon_{1} d \epsilon_{2} f_{\mathrm{F}}^{\prime}\left(\epsilon_{1}\right) f_{\mathrm{F}}^{\prime}\left(\epsilon_{2}\right) \\
& \times \int d \mathbf{x}_{1} d \mathbf{x}_{2}\left|P_{\epsilon_{1}, \epsilon_{2}}^{\Delta \phi}\left(\mathbf{x}_{1}, \mathbf{x}_{2}\right)\right|^{2} .
\end{aligned}
$$

Here $P^{\Delta \phi}$ is again given by Eq. (17) but now the continuous $\mathbf{q}$ have to be replaced ${ }^{25}$ by discrete momenta, $\mathbf{q}=\mathbf{q}_{m}(\Delta \phi)$ $=\frac{2 \pi}{L}\left(m+\frac{\Delta \phi}{\phi_{0}}\right)$, depending on the difference of the magnetic flux during the individual measurements of $g$. The fluctuations are a periodic function in $\Delta \phi / \phi_{0}$, where $\phi_{0}=2 \pi / e$ is the elementary flux quantum and $\Delta \phi=\Delta B L^{2} /(4 \pi)$. Therefore an expansion in its harmonics can be made, ${ }^{25}$

$$
\overline{\delta g(\phi) \delta g(\phi+\Delta \phi)}=\frac{C e^{4}}{\pi^{2}} \sum_{k=0}^{\infty} \mathcal{A}_{k}(B) \cos \left[2 \pi k \frac{\Delta \phi}{\phi_{0}}\right],
$$

where $C$ is a factor of order 1 , depending on the sample geometry in the vicinity of the ring. Restricting to the situation of strong spin-orbit scattering ${ }^{29}$ where the spin singlet diffuson gives the leading contributions to Eq. (19), one finds that (for $L \gg L_{\varphi}$ )

$$
\mathcal{A}_{k}(\mathbf{B})=\frac{(2 \pi)^{3} D^{3 / 2}}{T^{2} L^{3}} \int d \epsilon \frac{e^{-k L / \sqrt{D \tau_{\varphi}(\epsilon)}}}{\cosh ^{4}(\epsilon / 2 T)} \sqrt{\tau_{\varphi}(\epsilon)},
$$

where $\left[\tau_{\varphi}=\tau_{\varphi, S}^{(4)}\right.$ in the notation of Eq. (18)]

$$
\frac{1}{\tau_{\varphi}(\epsilon, T, B)}=\frac{2 n_{i}}{\pi \nu}\left(\pi \nu \operatorname{Im}\left[\mathrm{T}_{(4)}^{\mathrm{A}}(\epsilon, B)\right]-\left|(\pi \nu) \mathrm{T}_{(4)}^{\mathrm{R}}(\epsilon, B)\right|^{2}\right) .
$$

Here $\epsilon=\epsilon_{1}+\epsilon_{2}$ and we used that relevant contributions to the integral over energy differences, $\bar{\epsilon}=\epsilon_{1}-\epsilon_{2}$, result from energies $\bar{\epsilon} \ll 1 / \tau_{\varphi}$ to eliminate the $\bar{\epsilon}$ dependence. Notice that such a reduction to a single energy integral can only be done in a one-dimensional system where the $\mathbf{q}$ integral over the square of the diffuson, Eq. (17), is dominated by infrared diver- 
gences. In a $2-d$ system, e.g., relevant energies extend to $\bar{\epsilon} \sim T$. For the following, it is convenient to rewrite Eq. (22) [see also Eq. (10)] as

$$
\frac{1}{\tau_{\varphi}}=\frac{1}{\tau_{\text {hit }}} \frac{\left\langle\sigma_{\text {inel }}\right\rangle}{\sigma_{\max }},
$$

where $\sigma_{\max }=4 \pi / k_{F}^{2}$ is the cross section of a unitary scatterer, $\sigma_{\text {inel }} / \sigma_{\max }$ is the conditional probability of inelastic scattering if an electron hits the impurity, and

$$
\frac{1}{\tau_{\text {hit }}}=\frac{2 n_{i}}{\pi \nu}
$$

describes the typical "hitting rate."

As in the WL experiment discussed above, fits to experimental data have to be done with the $\epsilon$-independent dephasing rate. For a comparison with experiment we therefore have to give the $\epsilon$-independent dephasing rate which for $k=1$ is obtained by solving the equation

$$
\frac{L_{\varphi}(T, B, L)}{L} e^{-L / L_{\varphi}(T, B, L)}=\frac{3}{8 T} \int d \epsilon \frac{e^{-L / L_{\varphi}(\epsilon, T, B)}}{\cosh ^{4}(\epsilon / 2 T)} \frac{L_{\varphi}(\epsilon, T, B)}{L},
$$

where $L_{\varphi}=\sqrt{D \tau_{\varphi}}$ is the dephasing length. Notice that the actually measured dephasing rate depends on the length of the ring. It also differs from the WL result due to the different energy averages.

\section{NUMERICAL RESULTS FOR DEPHASING RATES FROM AHARANOV-BOHM OSCILLATIONS}

In order to evaluate the dependence of the dephasing rate on magnetic field, temperature and ring length from Eq. (27) we require the T-matrix for the single impurity Kondo model defined in Sec. II. By using the equation of motion method this can be expressed as ${ }^{30}$

$$
T_{\sigma}(\omega, T, B)=J\left\langle S_{z}\right\rangle+J^{2}\left\langle\left\langle\mathbf{S} c_{\alpha}^{\dagger} \boldsymbol{\sigma}_{\alpha \sigma} ; \mathbf{S} \boldsymbol{\sigma}_{\sigma \alpha^{\prime}} c_{\alpha^{\prime}}\right\rangle\right\rangle,
$$

where $\langle\langle\cdots\rangle\rangle$ denotes a retarded correlation function and $\boldsymbol{\sigma}$ are the Pauli spin matrices. We calculate Eq. (26) by applying the numerical renormalization group (NRG) method $^{31}$ for finite temperature dynamics. ${ }^{32}$ At finite magnetic field, it is also important to use the reduced density matrix ${ }^{33}$ to evaluate the above dynamical quantity. For all calculations presented here we used a discretization parameter for the conduction band of $\Lambda=1.5$ and we retained 960 states per NRG iteration. We checked that this number of states was sufficient to maintain particle-hole symmetry of the spectral densities $\operatorname{Im} T_{\uparrow}(\omega, T, B)=\operatorname{Im} T_{\downarrow}(-\omega, T, B)$ at this relatively small value of the discretization parameter. The Friedel sum rule for the $T=0$ spectral density was satisfied to more than $1 \%$ accuracy in our calculations.

Figure 9 shows the numerical evaluation of $\sqrt{\mathcal{A}_{1}(B)}$ for various $T$ at a given length $L=10 L_{\text {hit }}$ where $L_{\text {hit }}=\sqrt{D \tau_{\text {hit }}}$. Notice that, if lengths are measured in units of $L_{\mathrm{hit}}$, the amplitude $\left(A_{1} \tau_{\mathrm{hit}} T_{\mathrm{K}}\right)^{1 / 2}$ becomes a universal function of $B / T_{\mathrm{K}}$ and $T / T_{\mathrm{K}}$.

Figure 10 gives the magnetic field dependence of the

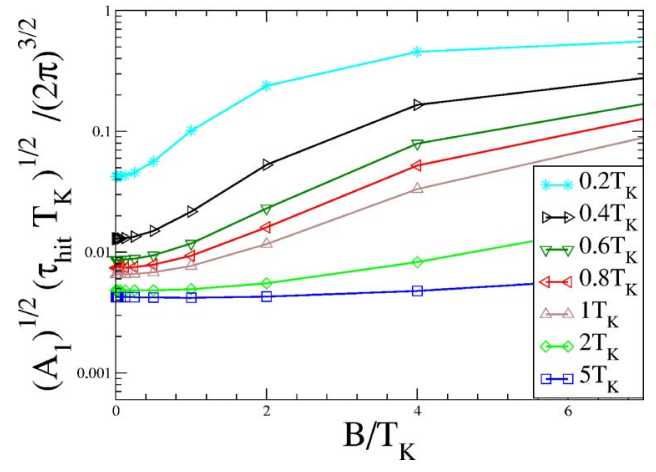

FIG. 9. (Color online) Amplitude of the Aharonov-Bohm oscillations (in units of $\left[(2 \pi)^{3} /\left(\tau_{\mathrm{hit}} T_{\mathrm{K}}\right)\right]^{1 / 2}$ ) as a function of the applied magnetic field, $B$ (in units $T_{\mathrm{K}}, \mu_{\mathrm{B}} / k_{\mathrm{B}}=1$ ), for different temperatures $T$ (in units $T_{\mathrm{K}}$ ) obtained from NRG calculations for $L / L_{\mathrm{hit}}=10$. The rapid rise of the oscillation amplitude results from the suppression of dephasing (see Fig. 10) by polarizing the spins. Here, and in the remaining figures in this section, the symbols represent the discrete values of $(B, T)$ at which NRG calculations were carried out.

dephasing rate at various $T$ and a fixed ring length $L=10 L_{\text {hit }}$. For large magnetic fields, $B \gg T, T_{\mathrm{K}}$, the dephasing rate is expected ${ }^{12}$ to vanish proportional to $(T / B)^{2} / \ln ^{4}\left[B / T_{\mathrm{K}}\right]$, consistent with the numerical results (the precise form also depends on $L / L_{\varphi}$, see below).

Figure 11 shows the results for $1 / \tau_{\varphi, S}$ as a function of $T$ for different strengths of the magnetic field $B$ at a fixed ring length $L=10 L_{\text {hit }}$. While the maximal dephasing occurs for $T \sim T_{K}$ for small fields $B \ll T_{K}$, it shifts to larger values $(T \sim B)$ for $B \gg T_{K}$. For high temperatures, small fields and not too large $L / L_{\varphi}$, see below, $T \gg T_{K}, B$ the dephasing rate is well described by the Nagaoka-Suhl formula, ${ }^{13}$ $1 / \tau_{\varphi}(T)=\frac{n_{\mathrm{i}}}{2 \pi \nu} \frac{\pi^{2} 3 / 4}{\pi^{2} 3 / 4+\ln ^{2} T / T_{\mathrm{K}}}$.

Figure 12 shows the dependence of $1 / \tau_{\varphi, S}(T)$ on the ring length, $L$, at zero magnetic field. As pointed out above, the $L$ dependence of the experimentally measured dephasing rate enters through the energy average of Eq. (25). As can be seen from Fig. 12 the dephasing rate only changes by a factor $1 / 4$ on increasing the ring length by a factor 200 . We included curves for the rather academic cases $L=100-1000 L_{\text {hit }}$ (the amplitude is too strongly suppressed to be observed for such

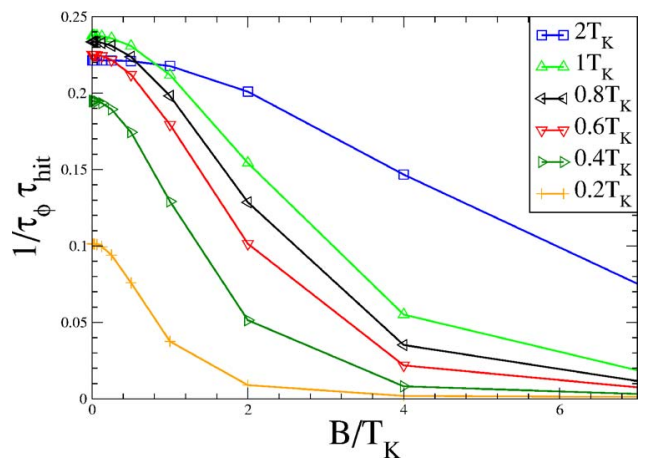

FIG. 10. (Color online) The dephasing rate as a function of the magnetic field, $B$ (in units $T_{\mathrm{K}}$ ) for various temperatures, $T$, and a ring of length $L=10 L_{\text {hit }}$. Note the rapid suppression of $1 / \tau_{\varphi}$ especially for low temperatures. 


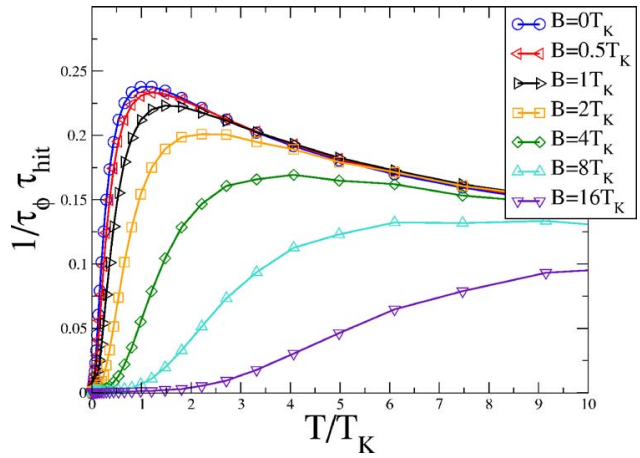

FIG. 11. (Color online) NRG results for the dephasing rate as a function of $T$ and for different values of $B . \tau_{\text {hit }}=\pi \nu / 2 n_{\mathrm{i}}$ as defined above, $T$ and $B$ are given in units of $T_{K}$ (Ref. 34) (we set $\left.\mu_{\mathrm{B}} / k_{\mathrm{B}}=1\right)$. The values are for a ring of length, $L, L=10 L_{\mathrm{hit}}$, where $L_{\text {hit }}=\sqrt{D \tau_{\text {hit }}}$. While the maximal dephasing occurs for $T \sim T_{K}$ for small fields $B \ll T_{K}$, it shifts to larger values $(T \sim B)$ for $B \gg T_{K}$.

large ring lengths) in order to show this weak $L$ dependence. Figure 12 also shows that for temperatures $T \leqq T_{K}$ the dephasing rate becomes entirely $L$ independent. This reflects the fact that the energy resolved dephasing rate, $1 / \tau_{\varphi, S}(\epsilon, T)$, establishes a deep minimum at $\epsilon=0$ for $T \leqq T_{K}$ and the integral on the right-hand side of Eq. (25) is therefore well approximated by setting $\epsilon=0$. To be precise, in the limit of ring lengths $L \gg L_{\varphi}$ the integral on the right-hand side of Eq. (25) (for fixed $T$ and $B$ ) is dominated by the saddle points of the function

$$
f(\epsilon)=\frac{L}{L_{\varphi}^{(4)}(\epsilon)}-\ln \left[\frac{L_{\varphi}^{(4)}(\epsilon)}{L}\right]+4 \ln \left[\cosh \left(\frac{\epsilon}{2 T}\right)\right] .
$$

For temperatures $T \lesssim \max \left\{B, T_{\mathrm{K}}\right\} f$ has a saddle point at $\epsilon=0$, which for temperatures $T \gtrsim T_{K}$ becomes unstable. At very large ring diameters $L / L_{\mathrm{hit}} \gg 10^{2}$, a second saddle point at $\epsilon=\frac{L}{2 L_{\mathrm{hit}} \ln ^{2}\left[L / 2 L_{\mathrm{hit}}\right]} T$ starts to dominate the integral for $T \gtrsim T_{K}$. Here $L_{\text {hit }}=\sqrt{D \tau_{\text {hit }}}$ is the diffusive length scale corresponding to the time $\tau_{\text {hit }}=\frac{\pi \nu}{2 n_{\mathrm{i}}}$ and introduced above. Although this limit is rather academic it is interesting that for such big rings dephasing is dominated by rare events of highly excited

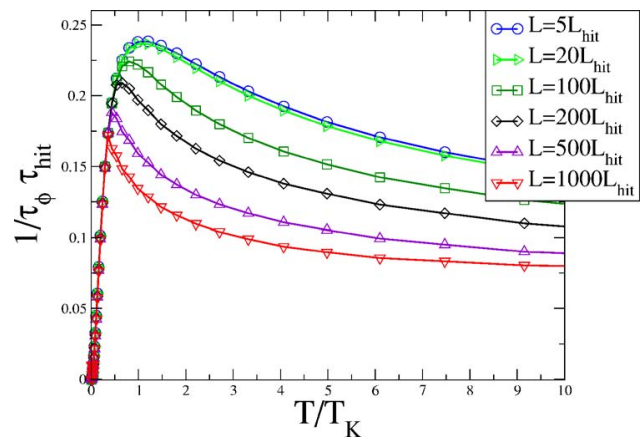

FIG. 12. (Color online) Temperature-dependent dephasing rate for various ring lengths $L$, measured in units $L_{\mathrm{hit}}=\sqrt{D \pi \nu / 2 n_{\mathrm{i}}}$ and calculated via NRG for $B=0$. The logarithmic suppression with $L$ for larger $T$ arises due to the interference of electrons with energies larger than $T$. thermal electrons scattering from the magnetic impurities. This originates from the fact that high-energy electrons scatter less effectively from Kondo spins, as Kondo renormalization becomes less effective for $\epsilon \gg T_{\mathrm{K}}$. Inserting this second saddle point into Eq. (25) one finds that the length dependence of $1 / \tau_{\varphi}$ for high temperatures follows

$$
\frac{1}{\tau_{\varphi}(T, L)} \sim \frac{1}{\ln ^{2}\left[\frac{L}{2 L_{\mathrm{hit}} \ln ^{2}\left[L / 2 L_{\mathrm{hit}}\right]}\right]},
$$

explaining the weak suppression of $1 / \tau_{\varphi}$ for large ring lengths shown in Fig. 12.

\section{DISCUSSION AND CONCLUSIONS}

In this paper we generalized previous results for the dephasing rate due to diluted Kondo impurities as measured in the weak localization experiment to describe dephasing due to arbitrary diluted impurities. Furthermore, we investigated how magnetic fields modify the dephasing rate due to Kondo spins as can be measured in mesoscopic AharonovBohm rings. We give results for the numerically evaluated dephasing rate as a function of the magnetic field, temperature, and the ring length.

The influence of magnetic impurities on dephasing has been studied in a number of magneto-resistance experiments

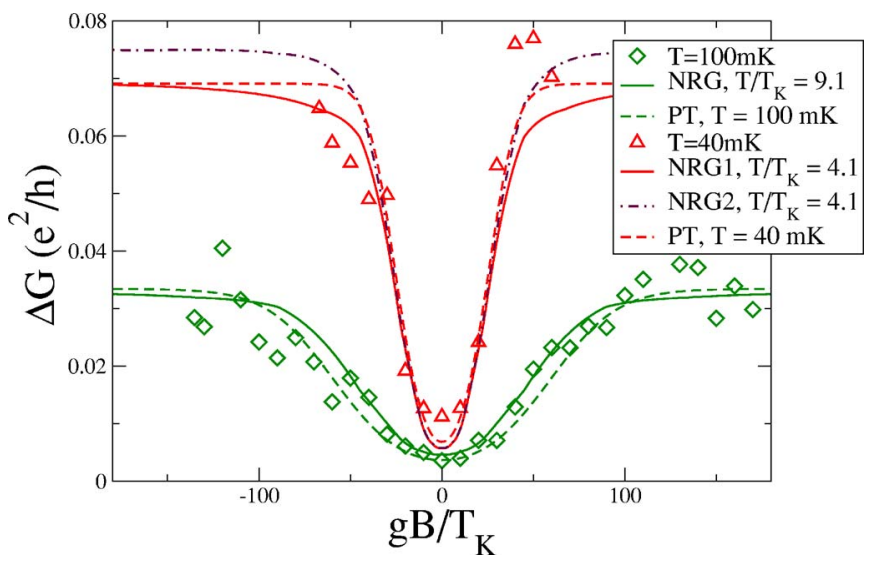

FIG. 13. (Color online) Amplitude of the $\mathrm{AB}$ oscillations in units of $e^{2} / h$ as a function of $T / T_{K}$ for $T=40 \mathrm{mK}(\Delta)$ and $100 \mathrm{mK}$ $(\diamond)$ measured by Piere et al. (Refs. 5 and 20), assuming $T_{K}=10 \mathrm{mK}$. Solid and dot-dashed lines are the numerically calculated amplitudes with fitting parameters described in the main text. As for very high magnetic fields, $B \gg 100 T_{K}$, numerical errors increase when the dephasing rate becomes very small, we used an extrapolation of the numerical results, $1 / \tau_{\varphi} \propto 1 / B^{2}$, in this regime. The saturation of the amplitude at these high fields arises as the dephasing due to electron-electron interaction dominates. The data is equally well described by the fits used in Refs. 5 and 20 (dashed lines), see main text. For the solid lines we used the same values for the dephasing rates $\left(\tau_{e e}=5.4 \mathrm{~ns}\right.$ and $9.9 \mathrm{~ns}$ for $T=100 \mathrm{mK}$ and $T=40 \mathrm{mK}$, respectively) as in Ref. 5, where $\tau_{e e} \propto T^{-2 / 3}$ was assumed. For the dot-dashed curve we use instead $\tau_{e e}=13.5 \mathrm{~ns}$ for $T=40 \mathrm{mK}$ since one expects theoretically ${ }^{35}$ that $\tau_{e e} \propto 1 / T$ for $L \gg L_{\phi}$ (note, however, that $L \sim L_{\phi}$ in this regime explaining the rather weak dependence on $\tau_{e e}$ ). 
in $\mathrm{Cu}, \mathrm{Ag}$, or $\mathrm{Au}$ wires doped with magnetic impurities. $2,3,5,15,16$ More recently, high-precision experiments using ion-implanted Fe impurities in Ag wires allowed a quantitative comparison with our theory for spin-1/2 impurities, see Refs. 15 and 16 for a critical discussion. Such studies using samples doped with magnetic impurities have, to our knowledge, only been performed in the spin-glass regime $^{36}$ using magnetic ions with tiny Kondo temperatures. Pierre et al. ${ }^{5,20}$ studied rings made from nominally clean $\mathrm{Cu}$ wires. As these wires show a saturation of the dephasing rate (determined from weak localization) at low temperatures ( $30 \mathrm{mK} \lesssim T \lesssim 1 \mathrm{~K}$ ), it was suspected that tiny concentrations of magnetic impurities with Kondo temperatures below $30 \mathrm{mK}$ may be at the origin of the observed saturation. This picture could be confirmed as measurements of the amplitude of Aharonov-Bohm oscillation displayed a dramatic rise by almost an order of magnitude in moderate magnetic fields (see Fig. 13), proving the magnetic origin of the low- $B$, low$T$ dephasing.

As neither the concentrations nor the type(s) of magnetic impurities are known, a parameter-free comparison to our predictions is not possible for these systems. Assuming Mn impurities, believed to be characterized by a Kondo temperature of the order of $10 \mathrm{mK},{ }^{37}$ and, using the same dephasing rates due to electron-electron interactions as in Ref. 5 ( $\tau_{e e}=5.4 \mathrm{~ns}$ and $9.9 \mathrm{~ns}$ for $T=100 \mathrm{mK}$ and $T=40 \mathrm{mK}$, respectively) we obtain the fits shown in Fig. 13 for a $g$ factor of $g \approx 1.4$ and an impurity concentration of $2.7 \mathrm{ppm}$. We have also added a curve at $T=40 \mathrm{mK}$ (dot-dashed line) which uses $\tau_{e e}=13.5 \mathrm{~ns}$ (keeping all other parameters identical) to take into account that one expects theoretically ${ }^{35}$ $\tau_{e e} \propto 1 / T$. The fits and the extracted parameters are not very reliable as can be seen from the observation that the data has been equally well described in Refs. 5 and 20 by the simple perturbative formula $\frac{\tau_{\varphi, S}(B=0)}{\tau_{\varphi, S}(B)}=\frac{g \mu_{B} / k_{B} T}{\sinh \left(g \mu_{B} / k_{B} T\right)}$ with $g=1.08$, see Fig. 13.

For a more meaningful comparison to our results, experiments on $\mathrm{AB}$ rings, doped with magnetic impurities with a higher Kondo temperature would be highly desirable. On the one hand, such experiments and their theoretical interpretation can reveal basic dephasing mechanisms in metals, on the other hand, they can be used to obtain insight into the physics of strongly correlated dynamical impurities and their interactions.

\section{ACKNOWLEDGMENTS}

We thank Ch. Bäuerle, N. Birge, J. v. Delft, L. Glazman, S. Kettemann, S. Mirlin, J. Mydosh, L. Saminadayar, B. Spivak, P. Wölfle, and especially, A. Altland for useful discussions and N. Birge for sending us his experimental data. Furthermore, we acknowledge financial support from the Deutsche Forschungsgemeinschaft through the SFB 608 and Transregio SFB 12 and the NIC Juelich for computing time.
${ }^{1}$ B. L. Altshuler, A. G. Aronov, and D. E. Khmelnitsky, J. Phys. C 15, 7367 (1982).

${ }^{2}$ P. Mohanty, E. M. Q. Jariwala, and R. A. Webb, Phys. Rev. Lett. 78, 3366 (1997); P. Mohanty and R. A. Webb, Phys. Rev. B 55, R13452 (1997).

${ }^{3}$ F. Schopfer, Ch. Bäuerle, W. Rabaud, and L. Saminadayar, Phys. Rev. Lett. 90, 056801 (2003).

${ }^{4}$ C. Van Haesendonck, J. Vranken, and Y. Bruynseraede, Phys. Rev. Lett. 58, 1968 (1987).

${ }^{5}$ F. Pierre, A. B. Gougam, A. Anthore, H. Pothier, D. Esteve, and N. O. Birge, Phys. Rev. B 68, 085413 (2003).

${ }^{6}$ D. S. Golubev and A. D. Zaikin, Phys. Rev. Lett. 81, 1074 (1998).

${ }^{7}$ I. L. Aleiner, B. L. Altshuler, and M. E. Gershenson, Waves Random Media 9, 201 (1999).

${ }^{8}$ J. v. Delft, cond-mat/0510563; J. v. Delft, in Fundamental Problems of Mesoscopic Physics, edited by I. V. Lerner et al. (Kluwer, London, 2004), pp. 115-138.

${ }^{9}$ A. Zawadowski, J. von Delft, and D. C. Ralph, Phys. Rev. Lett. 83, 2632 (1999).

${ }^{10}$ Y. Imry, H. Fukuyama, and P. Schwab, Europhys. Lett. 47, 608 (1999).

${ }^{11}$ P. Mohanty and R. A. Webb, Phys. Rev. Lett. 84, 4481 (2000).

${ }^{12}$ M. G. Vavilov and L. I. Glazman, Phys. Rev. B 67, 115310 (2003); M. G. Vavilov, L. I. Glazman, A. I. Larkin, ibid. 68, 075119 (2003).

${ }^{13}$ T. Micklitz, A. Altland, T. A. Costi, and A. Rosch, Phys. Rev. Lett. 96, 226601 (2006).
${ }^{14}$ G. Zaránd, L. Borda, J. von Delft, and N. Andrei, Phys. Rev. Lett. 93, 107204 (2004).

${ }^{15}$ F. Mallet et al., Phys. Rev. Lett. 97, 226804 (2006).

${ }^{16}$ G. M. Alzoubi and N. O. Birge, Phys. Rev. Lett. 97, 226803 (2006).

${ }^{17}$ The $h / 2 e-\mathrm{AB}$ oscillations arise by the same mechanism as the WL and are suppressed when the magnetic field penetrates the wire of the sample.

${ }^{18}$ A. Benoit, D. Mailly, P. Perrier, and P. Nedellec, Superlattices Microstruct. 11, 313 (1992).

${ }^{19}$ P. Schwab and U. Eckern, Ann. Phys. 5, 57 (1996).

${ }^{20}$ F. Pierre and N. O. Birge, Phys. Rev. Lett. 89, 206804 (2002); F. Pierre and N. O. Birge, J. Phys. Soc. Jpn. 72, 19 (2003).

${ }^{21}$ B. L. Altshuler and A. G. Aronov, in Electron-Electron Interaction in Disordered Systems, edited by A. L. Efros and M. Pollak (North-Holland, Amsterdam, 1985).

${ }^{22}$ S. Kettemann and E. R. Mucciolo, JETP Lett. 83, 240 (2006); cond-mat/0609279.

${ }^{23}$ S. Hikami, A. I. Larkin, and Y. Nagaoka, Prog. Theor. Phys. 63, 707 (1980)

${ }^{24}$ V. I. Falko, JETP Lett. 53, 340 (1991).

${ }^{25}$ A. G. Aronov and Y. V. Sharvin, Rev. Mod. Phys. 59, 755 (1987).

${ }^{26}$ I. L. Aleiner and Ya. M. Blanter, Phys. Rev. B 65, 115317 (2004).

${ }^{27}$ Notice that in the AB experiment (considered below) the suppression of inelastic vertex contributions is even larger and proportional to $1 /\left(\Delta E t_{\mathrm{D}}\right)^{2} \ll 1$ (Ref. 35), where $t_{\mathrm{D}}=L^{2} / D \gg \tau_{\varphi}$ and $L$ is the ring length. This is due to the fact that in this case the typical time scale of interfering electrons is $t_{\mathrm{D}}$ rather than $\tau_{\varphi}$ as elec- 
trons contributing the $\mathrm{AB}$ oscillations have to circle the ring at least once.

${ }^{28}$ R. A. Webb, S. Washburn, C. P. Umbach, and R. B. Laibowitz, Phys. Rev. Lett. 54, 2696 (1985).

${ }^{29}$ In their experiments F. Pierre et al. (Ref. 20) measured spin-orbit lengths of order $\sim 1 \mu \mathrm{m}$ much smaller than $L_{\varphi}$.

${ }^{30}$ T. A. Costi, Phys. Rev. Lett. 85, 1504 (2000); A. Rosch, T. A. Costi, J. Paaske, and P. Wölfle, Phys. Rev. B 68, 014430 (2003).

${ }^{31}$ K. G. Wilson, Rev. Mod. Phys. 47, 773 (1975); H. R. Krishnamurthy, J. W. Wilkins, and K. G. Wilson, Phys. Rev. B 21, 1003 (1980).

${ }^{32}$ T. A. Costi, A. C. Hewson, and V. Zlatić, J. Phys.: Condens. Matter 6, 2519 (1994).
${ }^{33}$ W. Hofstetter, Phys. Rev. Lett. 85, 1508 (2000).

${ }^{34} \mathrm{We}$ define and determine the Kondo temperature $T_{K}$ from the $T$ $=0$ suceptiblity $\chi=\left(g \mu_{\mathrm{B}}\right)^{2} /\left(4 T_{K}\right)$.

${ }^{35}$ T. Ludwig and A. D. Mirlin, Phys. Rev. B 69, 193306 (2004).

${ }^{36}$ J. Jaroszyński et al., Phys. Rev. Lett. 75, 3170 (1995); J. Jaroszyński, J. Wróbel, G. Karczewski, T. Wojtowicz, and T. Dietl, ibid. 80, 5635 (1998); G. Neuttiens, C. Strunk, C. Van Haesendonck, and Y. Bruynseraede, Phys. Rev. B 62, 3905 (2000); A. Benoit, D. Mailly, P. Perrier, and P. Nedellec, Superlattices Microstruct. 11, 313 (1992).

${ }^{37}$ D. K. Wohlleben and B. R. Coles, Magnetism, edited by H. Suhl (Academic, New York, 1973), Vol. 5. 